\title{
A Serious Games Platform for Cognitive Rehabilitation with Preliminary Evaluation
}

\author{
Paula Alexandra Rego ${ }^{1,2}$ (D) Rui Rocha ${ }^{3} \cdot$ Brígida Mónica Faria $^{2,4,5}$. \\ Luís Paulo Reis ${ }^{2,3,6} \cdot$ Pedro Miguel Moreira ${ }^{1,2}$
}

Received: 21 June 2016 / Accepted: 1 November 2016/Published online: 18 November 2016

(C) Springer Science+Business Media New York 2016

\begin{abstract}
In recent years Serious Games have evolved substantially, solving problems in diverse areas. In particular, in Cognitive Rehabilitation, Serious Games assume a relevant role. Traditional cognitive therapies are often considered repetitive and discouraging for patients and Serious Games can be used to create more dynamic rehabilitation processes, holding patients' attention throughout the process and motivating them during their road to recovery. This paper reviews Serious Games and user interfaces in rehabilitation area and details a
\end{abstract}

This article is part of the Topical Collection on Patient Facing Systems

Paula Alexandra Rego

paularego@estg.ipvc.pt

Rui Rocha

gdrui_fafe@hotmail.com

Brígida Mónica Faria

btf@estsp.ipp.pt

Luís Paulo Reis

lpreis@dsi.uminho.pt

Pedro Miguel Moreira

pmoreira@estg.ipvc.pt

1 ESTG/IPVC - Escola Superior de Tecnologia e Gestão, Instituto Politécnico de Viana do Castelo, Viana do Castelo, Portugal

2 LIACC - Laboratório de Inteligência Artificial e Ciência de Computadores, Porto, Portugal

3 DSI/EEUM - Departamento de Sistemas de Informação, Escola de Engenharia da Universidade do Minho, Guimarães, Portugal

4 ESS/PP - Escola Superior de Saúde, Politécnico do Porto, Porto, Portugal

5 INESC-TEC - Instituto de Engenharia de Sistemas e Computadores, Tecnologia e Ciência, Porto, Portugal

6 Centro ALGORITMI, Universidade do Minho, Guimarães, Portugal
Serious Games platform for Cognitive Rehabilitation that includes a set of features such as: natural and multimodal user interfaces and social features (competition, collaboration, and handicapping) which can contribute to augment the motivation of patients during the rehabilitation process. The web platform was tested with healthy subjects. Results of this preliminary evaluation show the motivation and the interest of the participants by playing the games.

Keywords Serious games $\cdot$ Rehabilitation $\cdot$ Cognitive rehabilitation $\cdot$ Natural user interfaces $\cdot$ Games $\cdot$ Health informatics

\section{Introduction}

As a multidisciplinary field of research, Serious Games have evolved substantially in recent years, solving problems in many diverse areas such as: military, education, rehabilitation and health care. Although there is no single accepted definition of the term, it has been consensus among the scientific community that Serious Games refer to games that have a specific main goal that goes beyond purely entertaining. In particular, in the field of Cognitive Rehabilitation, Serious Games are used as computer games whose main purpose is to reach a specific goal (rehabilitate) other than entertainment, and that use the entertainment component and the game's ability to hold the patient attention and interest while playing the game.

Several studies on rehabilitation show that the effectiveness is bigger when patients follow intensive training programs, oriented to reach a goal and divided into specific tasks that have to be performed repetitively $[1,2]$. The problem however with these traditional approaches is the lack of motivation and lack of interest of patients to perform these repetitive tasks. 
Rehabilitation professionals believe that the motivation of patients plays an extremely important role in the success of rehabilitation so keeping patients motivated throughout the process becomes crucial for more efficient rehabilitation. According to [3] (Maclean et al.), highly motivated patients realize more easily that rehabilitation is not only important, but in many cases is the only hope for their recovery and they are more willing to play an active role throughout this process.

To mitigate these problems, Serious Games-based tools are necessary to create more dynamic rehabilitation processes and allowing a different perspective of the traditional processes of rehabilitation therapy. These games should then be defined taking into account characteristics that are able to stimulate and train cognitive functions as well as features that require some kind of motor activity in order to stimulate and attract the attention of patients $[4,5]$.

The main objective of cognitive rehabilitation is the improvement of cognitive skills affected as a result of suffered brain damage. In this sense, it focuses mainly on returning patients to the maximum possible independence and the best possible functioning of their cognitive functions. These processes include cognitive faculties such as attention, concentration, memory, reasoning, problem solving, and language, among others. Serious Games can assume a relevant role in Cognitive rehabilitation. Despite literature review shows that Serious Games are most commonly used in physical rehabilitation therapy processes [5], several examples of serious games studies used for cognitive rehabilitation can be found. However, for most of these studies we found that they are still prototypes in development or in testing phase [6-9].

In a previous study we made a literature revision in Serious Games for cognitive rehabilitation and propose a set of features to be incorporated in the games [10]. Based on that, the main objective of this work is to highlight our work regarding the development of a web platform for Serious Games in cognitive rehabilitation that integrates the proposed features.

This paper extends our work presented in the WorldCIST'16 $-4^{\text {th }}$ World Conference on Information Systems and Technologies, in Recife, Brazil (Rui Rocha, Rego, Faria, Reis $\&$ Moreira, 2016). The rest of the paper is structured as follows. Second Section presents a characterization of the main modalities of interaction in cognitive rehabilitation games as well as its importance for rehabilitation and refers some of the main multimodal and natural interaction modalities present in these games. Third Section describes the importance of social features in rehabilitation games and Fourth Section presents a review of serious games for rehabilitation focused on the features we propose for designing games for cognitive rehabilitation. Following, Fifth Section describes the web platform for serious games, including the architecture, the integrated features and the developed games along with a brief description of the main features that were included in their implementation. Six Section describes the experiments with users and Seventh
Section presents results of user testing using the described architecture. Eight Section presents the major conclusions and some directions for future work.

\section{Natural and multimodal user interfaces}

Many of the patients who use rehabilitation therapies present various physical limitations in addition to cognitive difficulties [11, 12]. It is therefore essential to decrease the limitations of games with respect to usability and interaction with the use of user interfaces, as much natural as possible and according to a multimodal approach [13-16].

The multimodal term refers to the combination of multiple modalities, which in this particular case relates to the way the system receives its input / produces its output [17]. Thus, the multimodal approach is a combination of more than one interaction technology, such as input or output [18] that should serve as facilitator of the interaction between humans and the computer [13]. A multimodal interface can incorporate different combinations of voice, gestures and facial expressions, and other, more conventional, forms of interaction, such as mice and keyboards. The most common combination in the literature uses gestures and voice simultaneously [14]. Concerning the use of more natural forms of interaction, there is a collaboration between different modes of interaction, as a way to support the signal recognition process. For example, capturing the lip movements can help in the speech recognition process [13]. An architecture [7] that accommodates the inclusion of several modalities as modules and also some previous studies $[6,10]$ on usability are described in $[6,7,10]$.

Multimodal interfaces offer several advantages over more traditional user interfaces. Firstly, they provide a more natural and friendly user experience. For example, the Real Hunter System [19], an application designed to help users find houses, offers the possibility for users of pointing a finger at a house and make questions to the system about the house, using their voice [19]. Such combined and complementary interaction using gestures and voice illustrates the type of natural interaction experiences a multimodal approach can provide.

Similarly, a multimodal approach may be used in order to equip the system with redundancy which allows to open the use of the system to different people and in different circumstances [20]. The MATCHKiosk application [21], an interactive guide of New York, allows you to use voice or written input to perform searches on the city map. The users of such systems seem to prefer multimodal approach by the fact that the different types of modalities are more suited for different actions, thereby supporting each other and allowing alternative methods for people with different disabilities to interact with the system [22]. The use of these approaches is essential to develop systems for people with physical deficits, particularly regarding the movement of the upper limbs [23-25]. 
Natural user interfaces are a type of interaction between humans and the computer based on the analysis of natural human behavior. Human actions are interpreted by machines as commands that control system operations [26]. This means that humans are able to use the system with very little practice, through natural movements / actions, since these behaviors are deeply rooted in their daily experiments, reducing the cost of system use, time of learning, and time of getting used to the system commands, and making the interaction easier to handle and more intuitive $[6,27,28]$. The main objective in this approach is to interact with the computer the way we interact with the world [18]. For example, using hand gestures, head movements, body motions, or spoken commands to make a selection on the screen is more direct than using the keyboard, or the mouse. Using these interfaces the user can interact with the system without noticing that he is using an intermediary interface (when using the Nintendo Wii Wiimote, or the PlayStation Move motion controller, for example), and in many cases the interaction can happen when there is no contact with any type of physical device [27] (when using body gestures to interact, for example).

Any physical limitation that these patients may have must be attenuated by a fusion between a multimodal approach and the use of natural user interfaces. A multimodal natural approach enables an interactive environment that is more flexible, appealing, motivating and integrative through various interaction technologies. Relevant examples of the use of multimodal interfaces using the concept of an intelligent wheelchair can be found in [25, 28-34]. In these studies, the intelligent wheelchair is controlled using high-level commands in a multimodal interface that uses voice, facial expressions, head movements and joystick as the main input forms and users have the option to choose the input type that best fits their needs, thereby enabling them to increase their safety and comfort.

The vision-based technologies are the most frequently identified in the literature. An overview of this type can be described as the interpretation of any human behavior that can be recognized as a visual signal [13]. Among the various existing technologies we highlight: the analysis of facial expressions [35], detection of body movements [36], gesture recognition [37] and tracking of eye movements [38-40]. The use of newer and sophisticated technologies, such as biofeedback, capture motion sensors and haptic sensors may be particularly useful for users with large physical limitations.

In the next subsection we present in more detail a description of these technologies and some examples of their application.

\section{Analysis of facial expressions}

The analysis of facial expressions works with the recognition of image shapes of the human face, through a series of features observed by the movement / positioning of the eyes, eyebrows, nose and mouth, among others. Analysis of these characteristics may occur in two forms: static, when only the final state of a facial motion is analyzed; or dynamic, by monitoring the variation of these characteristics in time [41].

Besides being used as a modality, this technology can also be used as a behavior analysis tool. For example, it allows, among other things, the measurement of the users' satisfaction with the game, and can be incorporated to refine their level of satisfaction in the game [42]. One of the most commonly noted problems with this technology is that people hide and deliberately disguise their emotions in an attempt to not expose themselves [41], leading to recognition errors and misinterpretation of the commands used.

\section{Detection of body movements and gesture recognition}

The detection of body movement and gesture recognition [43] are the most common approaches in the literature and are usually used as a form of direct interaction with the system, in a perspective of a user command that triggers an action in the system [44].

The gesture recognition can be applied to the entire body [45], or may make use of specific gestures, such as head movements [46].

This technique is particularly useful in patients with reduced mobility and with limitations of the upper limbs.

More recent algorithms allow the use of this technique with common video cameras, and can be used as a form of direct interaction with the system. For example, using movements of the head to the right and left to make the user to move left or right in the game [46]. The most usual form of application of this technology is by the use of hand gestures. The recognition of these gestures may be achieved through the use of data gloves that allow to accurately record the bending of each finger, but this is a more intrusive approach which can cause discomfort to users [47]. This recognition can also be done through computer vision based interfaces, constituting a passive and silent recognition, and a non-intrusive technique, comparatively with the data glove methods, and providing thus a more intuitive way of communication between a human and a computer [47].

A study by Gerling et al. [36], in a group of elderly people with movement difficulties, examined the use of video games based on natural interaction, particularly full-body interaction, that is, using gestures performed with any body part. The interaction with the developed game was achieved through the use of the Kinect device [48] that through its RGB camera and depth sensor provides 3D capture capabilities of movements of the entire body [48]. In this study a game was developed using a series of pre-defined gestures: raise one arm, lifting both arms, clap, walking in the same place, say goodbye and remain standing supported only on one foot, among others. Special attention has been given in the 
definition of these movements so that most of them could be performed with the participants seated, due to their physical limitations. The results of this study indicate that the participants had a good experience at the fun level. Similarly, low levels of difficulty and fatigue were recorded during use of the game. From this study it is clear that gestures of hands and arms are easier to perform and learn, especially if they are already known gestures of daily actions (e.g. raise an arm). Some users found that the gestures were too simple and lacked any competition in carrying them.

The detection of hand and finger motions can also be done through the use of the Leap Motion sensor device [49] that is commercially available and constitutes another form of natural interaction with the computer. This controller plugs into a standard USB port and relies on built-in cameras and infrared LEDs to capture and analyze finger and hand movement. Leap Motion provides an extensive SDK for developers to incorporate support for the device into their own applications and supports several different programming languages across a number of platforms. Instead of developing the full mechanic for identification of the movement, with Leap motion the programmer can focus on the game, using the already existing techniques to detect hands movement.

\section{Tracking eye movement}

The use of the eyes as a way of interacting with computers has its origins in the analysis of gaze, that is, track to where a person is looking $[50,51]$. The analysis of gaze allows us to establish a link between the user and that to which he is paying attention [39, 52]. Based on this principle, Dickie et al. [53] developed two applications: a video player that automatically pauses when you look away from the screen, and a reading application of digital books that advances the text as the user progresses in their reading. Using the same principle, a similar system could be implemented in the games so that they could present some sound when the user looks away. In a multimodal perspective, this technology can be combined, for example, with voice recognition, or forms of more traditional interaction, such was illustrated in the system described by Zhai et al. [54] where the mouse cursor displacement was achieved through the gaze, while the actions of click and selection continued to be performed with the mouse.

Developments in this area now allow tracking of eye movements, allowing people with movement difficulties, especially at the level of the upper limbs to interact with computers, for example using the eyes to move the mouse cursor and blink an eye as a way of clicking [52]. Although it is common to capture these signals by similar devices to video cameras, it is possible to integrate these systems into a pair of ordinary glasses [55].

Being concentration one of the versed modalities in cognitive training, this technology could prove equally useful in this type of games, for example, to analyze which elements of the game users pay more attention.

\section{Audio-based technologies}

The audio-based technologies deal with information from several sound signals. Although the nature of these signals is not as diverse as visual signals, the acquired information may be more reliable and useful [13]. Several technologies emerge under this modality.

Historically, speech recognition [56] has given prominence by researchers. Scientific research related to the treatment of audio signals have been working in what is one of the biggest challenges of speech recognition, the development of systems capable of operating well in noisy environments. For that purpose, technological advances have focused on improving the accuracy and the ability and the ability of systems to give meaning to the words in the natural language of human [56].

The voice recognition consists in interpreting voice commands dictated by the user to be used as a system control method [57]. For several years the audio-based technologies deal not only with voice recognition, but make also use of other sounds made by the human vocal apparatus than voice such as whistles, whispers, groans and hums, among others [58, 59]. This form of interaction with the systems has proved effective with regard to the control of the mouse cursor [60] and in emulation of keyboards [59, 61], as well as a form of interaction in games [62, 63]. Sporka et al. [63] adapted the common Tetris game to take both verbal commands and non-verbal. Verbal commands passed simply by users say "left" to move the pieces to the left or "right" to move to the right, while nonverbal commands are characterized by a kind of groan ("hmmm") that with a higher tone shifts the pieces to the right and a lower tone would shift the pieces left. Overall the study participants found the intervention methods much more fun than by using typical keyboards and commented that these methods would be greatly appreciated by users with mobility difficulties. Results suggested that non-verbal controls were shown to be more accurate and likely to execute faster.

The attempt to integrate human emotions human-computer interaction led to the analysis of emotions through voice [64, 65], another field of research in the area of capture and analysis of audio signals. What this technique aims to do is to automatically identify, from the voice, emotional or physical state of the user [65]. You can identify emotions from annoyance, anger, boredom, fear and indifference, among others [65]. This technology is currently used for example in reservation systems of automated tickets, scheduled to see if the user shows boredom or frustration, and changing the answer in function of that $[65$, 66]. There are also studies in medicine, where therapists apply this technology in the detection of signs of depression and suicide risk [67, 68]. Also other non-verbal sound signals, typically human, as sighs have been analyzed in order to improve 
the analysis of emotions through audio [69]. A study of Kostoulas et al. [70] describes the implementation of a speech interface, as part of a platform for the development of Serious Games (PlayMancer platform) for patients with mental disorders, which has two components: voice recognition and recognition of emotions through voice. For both components positive results were obtained in terms of performance.

\section{Motion capture systems, haptic technology and biofeedback}

The use of newer and sophisticated technologies, such as biofeedback, capture motion sensors and haptic sensors may be particularly useful for users with large physical limitations.

Motion capture systems are a technology widely used in film, animation and video games. Many are based on suits worn by users [13], which let to capture all the movements made by them. This technique besides being invasive becomes unsuited for individuals with physical limitations.

The forms of interaction with a system must take into account the response of the system itself, or the system outputs and thus enable users to receive system information also through various methods, for example by sound effects and graphics. The haptic technology is an example of output feedback that is based on tactile feedback, through the application of forces or vibrations to users [71]. This technology is often used in robotics and virtual reality. Current humanoids robots have this technology in order to make them aware and sensitive to the touch [72]. Force-feedback can contribute to increase the realism of the game and make the user/patient closer to the environment of the game.

Biofeedback is a process that uses the physiological signs of individuals in order to manipulate and use them as a way of interacting with a system $[73,74]$. There are two perspectives as regards the use of biofeedback-based technologies. On the one hand, physiological signals indirectly controlled by the user such as heartbeat and brain waves can be used. This perspective therefore refers to signals that cannot be explicitly influenced by the user and as such do not make up an effective interaction with the systems, due to the fact that are very restrictive in terms of gaming experience. On the other hand, the use of physiological signals controlled directly by the users (e.g. the flexure of muscles or breathing patterns) can be effectively used as a means of interaction, especially in people with difficulty in moving themselves [75]. This approach uses several bio-sensory technologies such as electroencephalography, electromyography, electrocardiography, measurement of respiratory changes and temperature sensors, among others [76]. Since we are dealing with patients, and trying them to surpass themselves, they are very likely to get excited. So, we should consider the controlling of their vital signals. As an example, the cardio fitness machines control the heart rate.
Use of natural and multimodal interfaces in serious games

The literature review allows to realize that vision-based technologies are the most common forms of natural interaction with video games and present good results regarding user experience and ease of getting used to the system.

Several studies have been conducted on the use of these technologies in Serious Games. Conconi et al. [74] studied the applications of movement detection and facial expression recognition, Flynn et al. [77] worked with tracking technologies of eye movements, Moussa et al. [35] tested the voice recognition while Saposnik and colleagues [78] conducted experiments with motion detection. Large companies of video games are increasingly focusing on these forms of interaction and these are increasing on the market. The Microsoft Kinect [48], the Leap Motion controller [49], the Nintendo Wii [79] and the Sony PlayStation Move [80], are just some of the devices commercially available.

\section{Social features}

A patient committed with his rehabilitation program increases his chances of success. Motivation plays an essential role during rehabilitation therapy. The encouragement of patients throughout the rehabilitation process shows up crucial in achieving the objectives. For this purpose, it is essential to find a balance between work and entertainment, and it is necessary to make the therapy most fun, entertaining, appealing and additive.

Social interaction in video games showed positive effects in tests with healthy subjects, with respect to the gaming experience [81]. In this test the participants were 42 graduate and post graduate students, aged between 16 and 34 and played an arcade-like game in competitive mode using three alternated configurations in terms of social context: virtual co-player setting (participants were told they played against the computer, but they played against their partner, in separate rooms), mediated co-player setting (the pairs played online against each other in separate rooms) and co-located co-player (they played against each other in the same room and in the same console). Results showed that co-players contributed in a positive way to the player's involvement in the game. The same can be studied with patients in rehabilitation through social interaction features incorporated in serious games, including collaboration and competition, making it possible to increase the degree of motivation and commitment of patients in the continuous practice of the exercises they have to perform [11].

In a previous study we made a literature revision on Serious Games for cognitive rehabilitation and proposed a set of social features to be incorporated in the games [10] such as: collaboration, competition and handicapping. 
Through collaborative features, interaction between patients is promoted, the social bond is increased and a fresh gaming experience is provided. The goal is to form a team and patients to be able to act as such, serving to constantly support and being a source of motivation to other patients in the team. Although not yet a much discussed topic, several studies in this direction have already been made [81-83].

The competition features call for the competitive spirit of the patients. Here the goal is not to form teams but to play against adversaries. Two patients at the same level of recovery, or similar limitations are confronted by the best result.

However, a key aspect to take into account when it comes to competition is the disparity between patients. Different patients have different limitations and are at different stages of rehabilitation and is not always easy to find two patients with the same type of limitations or in the same rehabilitation stage for playing together. However, it is important to create a way in which all these patients may play and interact in order to maximize the interaction and gameplay possibilities. Thus, it becomes essential to guarantee equal conditions for all participants. In this perspective, follows the handicap concept, or handicapping. Handicapping is a concept used in games and in certain sports as a way to match / even the probabilities for all participants win by granting advantages in the form of a one-off compensation or any other benefit granted to participants at a distinct disadvantage. The normal situation when employing this technique is to award a disadvantage to the most experienced player to allow the player with less experience to participate in the game, or sport in a fairly way. This technique is widely used in golf, chess, bowling, horse racing and polo, among others.

\section{Serious games for cognitive rehabilitation}

Serious Games can be applied in several areas, from military training programs to the educational field. In this research we focus the application of serious games in cognitive rehabilitation therapies.

Several studies that address the use of serious games in the area of cognitive rehabilitation have already been conducted $[74,84,85]$.

In Conconi et al. [74] the authors present the PlayMancer platform in order to develop serious games in a quickly way, focused on behavioral disorders and addiction. This platform includes a multimodal interaction interface (voice, touch, biosensors and motion capture). The goal of this game was to increase problem-solving skills of patients, improve selfcontrol and impulsive behaviors.

Caglio et al. [84] created a 3D driving simulator in order to understand the changes that occur in cognitive functions (spatial and verbal memory) in patients suffering from Traumatic Brain Injury.
Cameirão et al. [85] developed a game based on motion capture with a camera for victims of stroke and traumatic brain injury. This game combines the characteristics related to motor and cognitive functions. The system uses the movements captured to activate intact neuronal systems that provide direct stimulation to the motor areas affected by brain injury.

Table 1 presents a comparative analysis of some of the serious games application analyzed in a revision of the literature. This analysis is focused on the following criteria: application area, use of a multimodal approach, user interaction technology used, number of players, and implementation of social features.

By analyzing Table 1, of the eleven studies reviewed, only three focus exclusively on cognitive rehabilitation and address both physical and cognitive abilities. Although there is a certain consensus on the use of natural interaction interfaces (only two studies do not use any variation of these interfaces), the vast majority of these studies did not include a multimodal approach, allowing only one way of interacting with the games. Similarly, it is inferred that the capture / detection of movement is the most common interaction technology in these studies.

Only three of these studies focus on the theme of a social approach to the game and only the study of Alankus et al. (2010) [11] addresses simultaneously competition, collaboration and handicapping.

It is assumed therefore crucial to further investigate a multimodal approach to forms of interaction in the games and the incorporation of social characteristics.

Besides the aspects mentioned, there are limitations associated to the games themselves that can be referred from the reviewed studies and that can be explored to improve rehabilitation games. These limitations can be associated, for example, with the quality and richness of the narrative, audio and graphics used, or the difficulty levels that the game provides which can thus provide more or less involvement (immersion, engagement, and sense of balance between skills and challenges) of the patient in the game. The tasks in the games should be meaningful to the patient. Most of the reviewed games were simple in terms of the narrative, graphics and feedback information provided, not exploiting most of the entertainment characteristics that games can provide.

This reviewed studies comprise only prototypes under development and testing phase. However, there are already some commercial systems used in hospitals and rehabilitation clinics, such as Rehacom [86, 87], Parrot Software [88] and Gradior cognitive software from INTRAS Foundation [89].

\section{Rehab+ platform}

A Web platform comprising a set of games adapted for use in cognitive rehabilitation has been developed. In this way the 


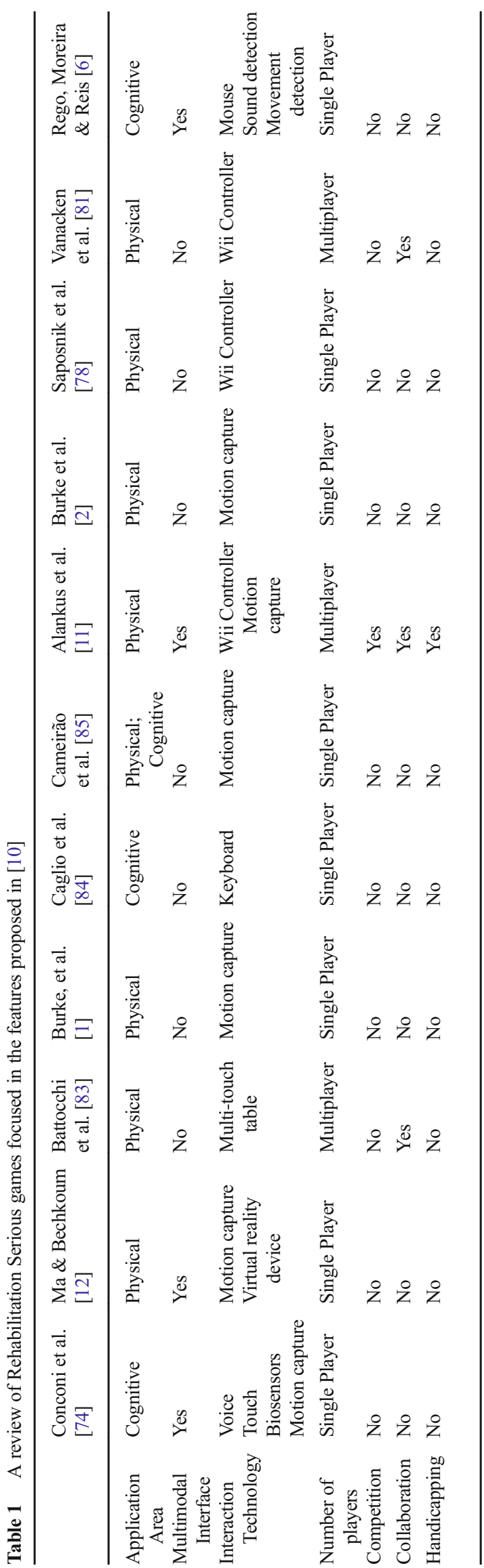

game can be played online, making it more accessible to all users, including patients in rehabilitation. Besides that, the web platform provides a low cost solution to patients training and eases a home rehabilitation, in addition to traditional therapy. In this section we describe the solution architecture of the Rehab+ platform, the games that are part of it, the technologies used in its creation and the tests carried out in the validation.

\section{Solution architecture}

The design of rehabilitation characteristics is a complex task. First of all, because it depends on the limitations of each patient but additionally because it must be made effective by a multidisciplinary team composed of professionals from various fields such as psychologists, physicians, and therapists, among others.

The first step in the development of Rehab+ consists in defining features to be included in each of the games developed. In this work, we decided to develop the games of the Rehab+ platform with similar rehabilitative features to an existing and established set of rehabilitation games that compose the RehaCom software [86, 87], and provide the Rehab+ games with the features proposed in our investigation. The RehaCom software, used for computer-assisted cognitive rehabilitation in many hospitals and rehabilitation centers around the world, provides a set of games, divided in training modules, to improve the cognitive functions (such as attention, concentration, memory, perception, activities of daily living, among others).

It was decided that each game should contain rehabilitative features capable of stimulating at least one cognitive function, as well as some of the features we proposed in this research as a way to improve the motivation of patients in rehabilitation. These features include the use of natural interaction interfaces, the use of social features as competition and collaboration, and the introduction of a handicapping mechanism.

Additionally, in order to verify the existence of different reactions of individuals when faced with games that provide the proposed features, and other games that do not offer them, we decide to develop a game that does not provide any of the proposed features, and another version of the same game that includes some of them.

The Rehab+ platform comprises all the games developed during this work, providing the solution that integrates all of the games in one place. This platform groups the games in two categories: single player and multiplayer. Figure 1 presents the architecture diagram of the Rehab+ platform, providing an overview of the integration of the proposed features in several developed games.

Eight games were developed that include different approaches to the same game: memory, sorting, arithmetic and "Tic-Tac-Toe" (or noughts and crosses game). For example, 


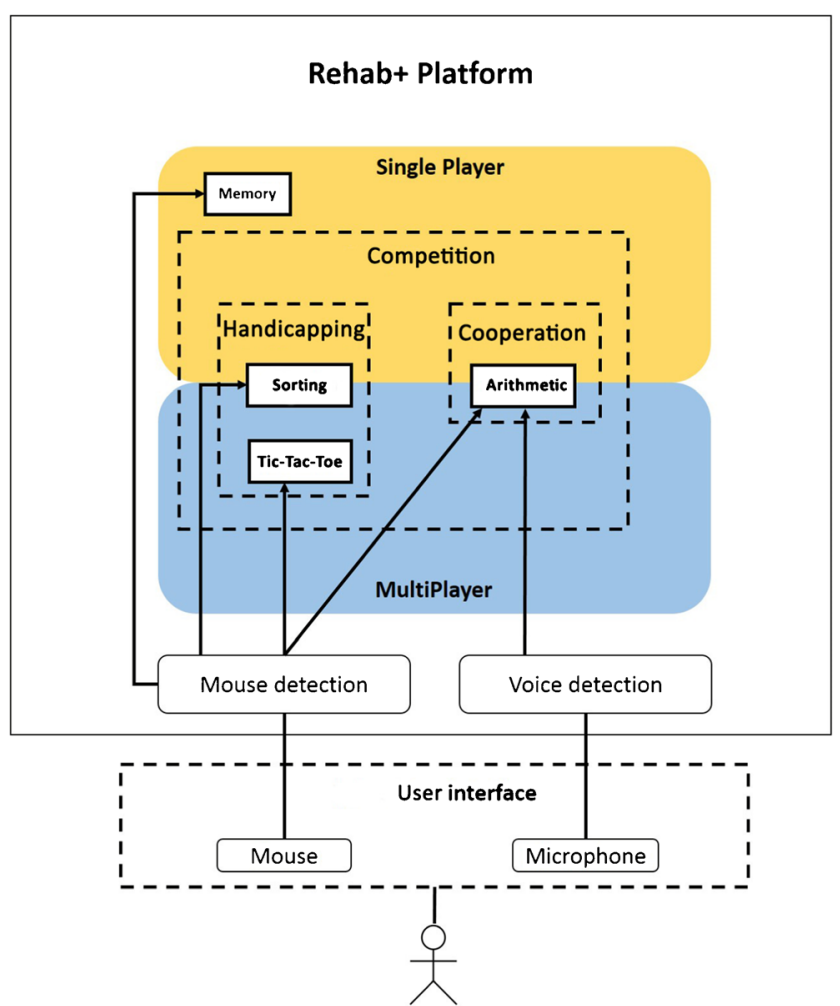

Fig. 1 Architecture diagram of the Rehab+ Game platform

in the single player category/approach the games included are: memory, sorting, arithmetic (using mouse) and arithmetic voice (arithmetic using voice commands) and in the multiplayer category/approach the games included are: "Tic-TacToe" handicap ("Tic-Tac-Toe" with handicap mechanism), arithmetic COOP (arithmetic using a cooperative approach), sorting multiplayer, and sorting handicap (sorting using handicap mechanism).

The memory game does not include any of the proposed features and is only available in single player mode. The sorting game, available in single player and multiplayer, covers competitive features and a handicapping system. The arithmetic game is available both in single player, and in multiplayer version, the later version being based on cooperative features, and the single player version also has the possibility of interaction using voice recognition. In the "noughts and crosses" game it was implemented a handicapping system. All the games have interaction via mouse.

\section{Integrated features}

In this investigation several innovative features were proposed whose main objective is to increase the motivation of patients during the rehabilitation process.

These features were designed to make games more motivating, attractive and easy to interact, by using a natural interaction interface and social characteristics, in particular: the competition, cooperation and the concept of handicapping.
In the games developed for this research we tried to incorporate in the best possible way, the proposed features to meet the initial objectives. And as such, these games include all the features proposed.

The games developed follow a competition and collaboration approach, and include a natural user interface to control the game (in this case, using voice commands). It was also implemented a handicapping system in two games.

User interface All games developed enable interaction via a mouse or via a touch screen, if they are used in mobile devices. The use of this type of games should foresee possible motor difficulties for users. In this sense, it was included in one of the developed games an alternative mode of interaction. Through the use of a JavaScript library and the Google Chrome browser it is possible for users to interact with the game "arithmetic voice" through voice commands, thus adapting the game to different profiles of users with varying abilities and difficulties/limitations and thus exploring the concept of multimodality. This form of interaction by voice commands also requires the use of a microphone. In this work various types of microphones were tested, and similar results have been obtained. This type of natural user interface also allows users to interact with the system without the need to use an external device to the computer, since the built-in microphone on most laptops is sufficient to ensure a stable interaction. The major limitation of this user interface relates to its use in environments with a lot of background noise, making it difficult a correct detection of the necessary voice commands. In addition, it requires the use of a browser that allows voice interaction, which limits the choices for users and may require the installation of specific software. However, of the most used browsers on the market, Google Chrome is the only one to allow this type of interaction, and is also at the date of writing this document, the most used in the world [90]. Also to attenuate this obligation for using the Google Chrome browser is the fact that all the various existing browsers currently have a very similar operation, which does not require a major effort for learning and getting used to it.

Cooperation Cooperation characteristics are part of a social approach to this kind of games, which aims to promote increased interaction between patients. Our proposed hypothesis is that this type of features enables patients to increase their motivational levels in relation to games and the rehabilitation process, from the companionship of their peers that are using the same games they too use. In this particular case, it is expected that, due the fact that patients are playing as a team, they will interact more, communicate more with their peers and create social bonds with their teammates, trying to avoid their isolation. These features were incorporated into the arithmetic game and were designed so that each user has a role 
Table 2 Rehab+ games characteristics

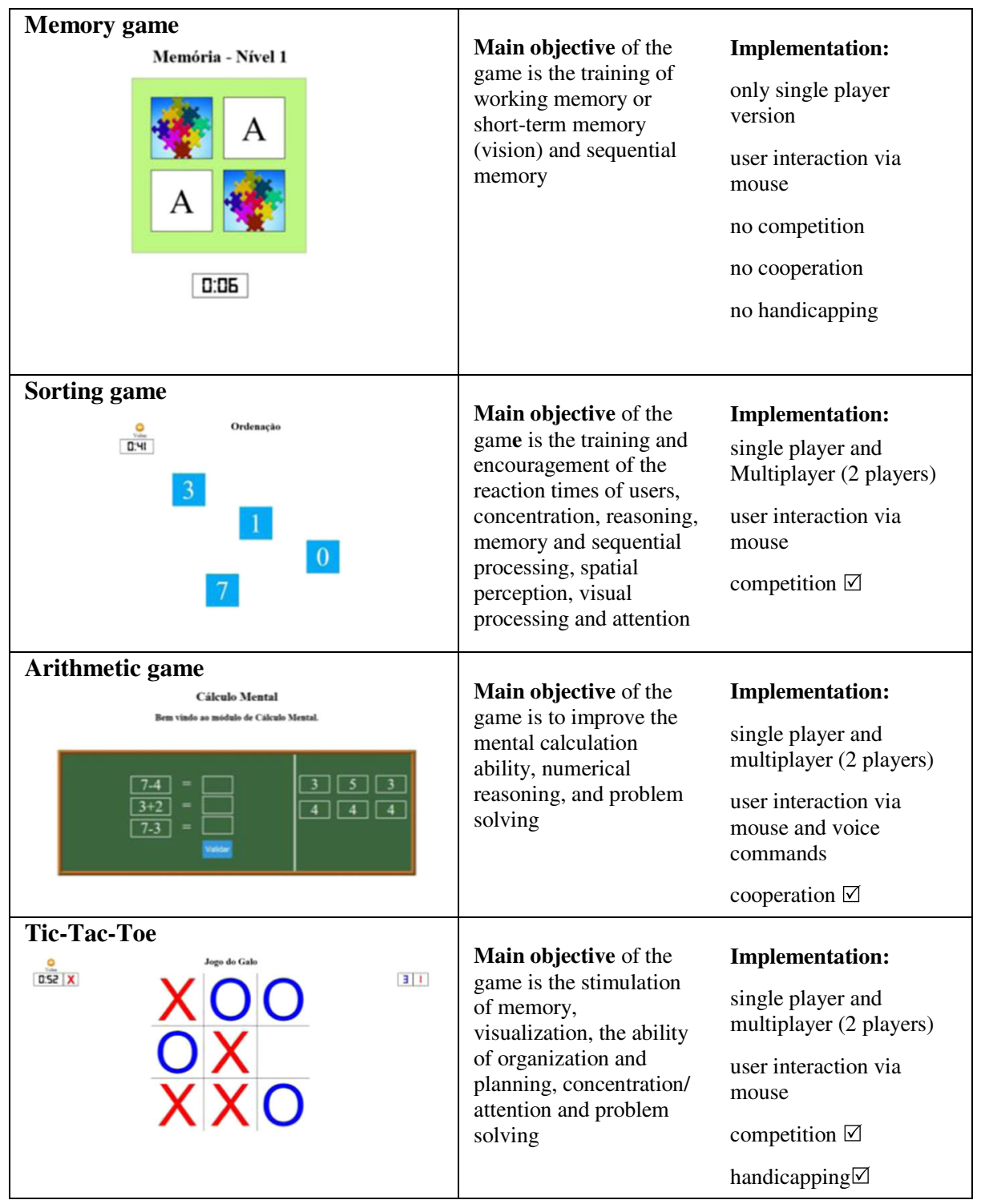

in the completion of the task. Thus, each user plays a specific role to be played so that both can attain success as a team.

Competition and handicapping A competitive game approach exploits similar concepts to those of collaboration, in the extent that is expected that patients will be motivated by sharing their experience with their peers. The characteristics of competition assume that two users are confronted for reaching the best individual result, not acting as a team but as opponents. It is therefore important to address the disparity difficulties of several patients. Different patients can have different problems and different types of limitations, and therefore when they confront themselves in these conditions, it is complicated, unfair and not motivating for them, which would prove to be

Table 3 Samples characterization of classroom and online tests

\begin{tabular}{|c|c|c|c|c|c|c|c|}
\hline \multicolumn{4}{|l|}{ Classroom test } & \multicolumn{4}{|l|}{ Online test } \\
\hline $\begin{array}{l}\text { Gender } \\
\text { (Females; Males) }\end{array}$ & $\begin{array}{l}\text { Age } \\
\text { (mean; std) }\end{array}$ & $\begin{array}{l}\text { Motor difficulty } \\
\text { (yes; no) }\end{array}$ & $\begin{array}{l}\text { Education Level } \\
\text { (High School/BSc/ } \\
\text { MSc/PhD) }\end{array}$ & $\begin{array}{l}\text { Gender } \\
\text { (Females/Male)s) }\end{array}$ & $\begin{array}{l}\text { Age } \\
(\text { mean/std) }\end{array}$ & $\begin{array}{l}\text { Motor difficulty } \\
\text { (yes/no) }\end{array}$ & $\begin{array}{l}\text { Education Level } \\
\text { (High School/BSc/ } \\
\text { MSc/PhD) }\end{array}$ \\
\hline $6 ; 16$ & $27 ; 9.2$ & $1 ; 21$ & $4 ; 8 ; 10 ; 0$ & $11 ; 25$ & $25 ; 6.9$ & $0 ; 36$ & $4 ; 18 ; 12 ; 2$ \\
\hline
\end{tabular}


Table 4 SUS and IMI results by classroom and online tests

\begin{tabular}{llll}
\hline Classroom test & \multicolumn{3}{c}{ Online test } \\
\hline SUS score & IMI score & SUS score & IMI score \\
$90.2 ; 9.5$ & $90.9 ; 9.1$ & $87.1 ; 12.0$ & $83.0 ; 13.5$ \\
\hline
\end{tabular}

contradictory to our purpose of the inclusion of these features. Thus, the concept of handicapping can be used in these situations since it seeks to equalize opportunities to win for each user. For example, in the multiplayer version of the sorting handicap game, system checks of the levels at which each user is are performed. If the user is playing a level above his opponent, the system makes the numbers to sort a second longer to appear than the numbers of his opponent. When the level difference is greater than two, the user that is in advantage receives only half of the bonus value whenever he completes a level.

Table 2 presents a list of the developed games, presenting for each game some of their characteristics: graphic interface, main objective of the game in rehabilitation terms, interaction technology used and social features that were implemented.

\section{User testing}

The tests described here were designed primarily to evaluate the usability and attractiveness of the games, to see if they are easy to play and interact, if it is easy to understand what is asked in each of them, if users like the games and if these are appealing.

The user tests counted on a total sample of 58 subjects, 36 took part in the online test and 22 participated in tests in the classroom. An initial pre-test was done in classroom, with nine subjects in order to finalize the details of the tests and games. After this pre-test, some errors detected in the games were corrected. Another objective was to verify if there were different opinions about the usability and attractiveness in the groups that played online or in the classroom.

It was developed a questionnaire that was applied to all subjects who participated in the test. In this questionnaire questions regarding multiplayer experiences were drawn for the participants online. The questionnaire has an initial section which aims to make a characterization of the sample (age, sex, and education, among others). Also it uses two validated instruments, commonly used in testing computer games: IMI (Intrinsic Motivation Inventory) and SUS (System Usability Scale). In the end, the questionnaire includes some questions that specifically address a comparison between the games modes and forms of interaction and an open response space to collect any comments that users want to express.

IMI is a multidimensional assessment tool that evaluates the subjective experiences of the participants in relation to the activity they experienced which, in this case, is in relation to all the gaming experience of activity and interaction with the Rehab+ platform. We adapted this instrument in order to use only one of their ranges, specifically the scale that measures the interest / satisfaction of the subjects in relation to the whole experience. The analyzed questions were: 1 . I enjoyed doing this activity very much; 2 . This activity was fun to do; 3 . I thought this was a boring activity; 4 . This activity did not hold my attention at all; 5. I would describe this activity as very interesting; 6. I thought this activity was quite enjoyable; 7 . While I was doing this activity, I was thinking about how much I enjoyed it.

The SUS is an instrument composed of 10 questions used to assess the usability of various products and services, hardware, software, among others. The questions in this instrument have been adapted to refer to the whole experience of interaction with the Rehab+ platform and the games that are part of it. The SUS items are: 1 . I think that I would like to use this system frequently; 2. I found the system unnecessarily complex; 3 . I thought the system was easy to use; 4 . I think that I would need the support of a technical person to be able to use this system; 5 . I found the various functions in this system were well integrated; 6 . I thought there was too much inconsistency in this system; 7. I would imagine that most people would learn to use this system very quickly; 8. I found the system very cumbersome to use; 9. I felt very confident using the system; 10 . I needed to learn a lot of things before I could get going with this system.

The tests were performed according to two different approaches: in classroom and online. The online tests contemplated only games in single player version, since it is impossible to
Fig. 2 SUS answers distribution - classroom test

\section{SUS - classroom test}

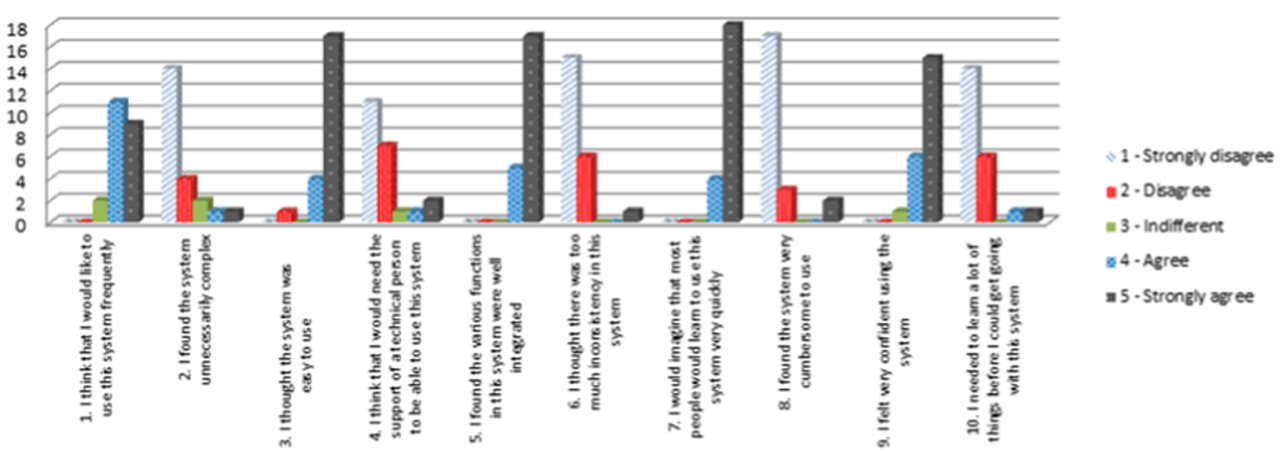


Fig. 3 SUS answers distribution - online test

\section{SUS - online test}

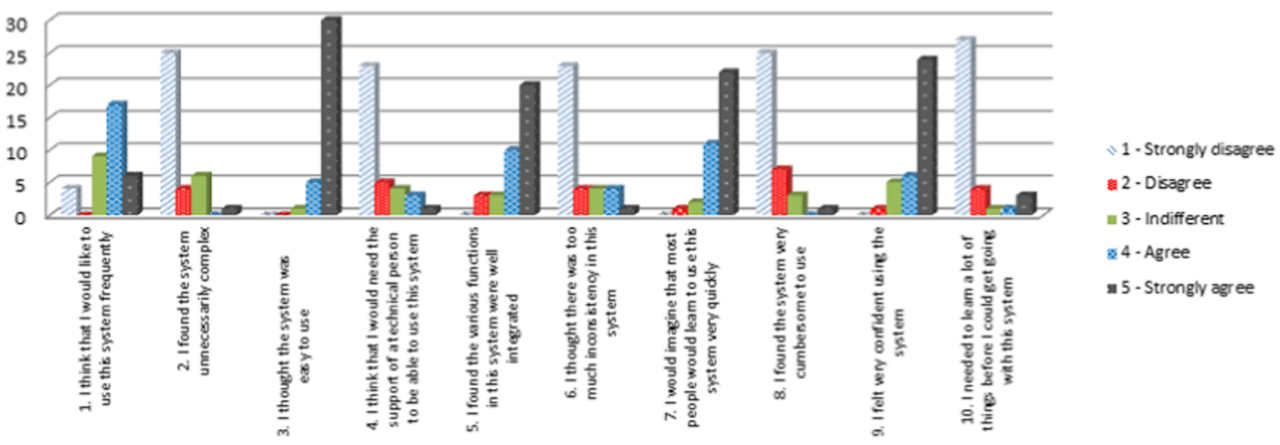

control whether users actually played the games with other users or not, and also because for these games it is required a specific software for allowing the use of simultaneously two independent mice in the same computer. In this approach, each of the games has a small description that clarifies about the goal and the rules of the game and how to play it.

In the tests realized in the classroom, subjects started to play alone all single player games, and then were grouped in pairs to test the multiplayer games. The tests began with a short explanation of the research and about the purpose of these games. Then each subject read and signed an informed consent, which contained all the information regarding the research and testing in which they would participate.

In what concerns the arithmetic game it was not defined any specific order for testing the two modes of interaction, which resulted in some participants experiencing first the interaction via mouse and others via voice commands.

In relation to the multiplayer version of the sorting game, all subjects experienced first the normal version and then the version with handicapping. It was not explained to the subjects nothing about the handicapping system before they played the games. In the questionnaire, all subjects were asked about the handicapping system, and if they noticed its existence during the game and all answered they did not notice.

It was shown for each subject how to interact with each game, and each one played the games during about 15-20 min before completing this questionnaire.

\section{Results and discussion}

The questionnaires were analyzed using the IBM SPSS Statistics software, in which were inserted all the answers and the statistical analysis was performed. It was considered 0.05 for the significance level. Statistical measures such as mean and standard deviation were applied to characterize the sample; and Mann-Whitney test was used to verify differences between two independent samples, namely the difference between the classroom and online tests and to verify the differences between the considered groups of age ( $<30$ years, $>30$ years).

Table 3 presents the characteristics of the samples (of the classroom and online tests) and mean and standard deviation of the respective age.

The SUS questionnaire was used to evaluate the usability of the whole platform. The SUS instrument is quoted from 0 to 100. A score below 70 means that the system presents
Fig. 4 IMI answers distribution classroom test

\section{IMI - classroom test}

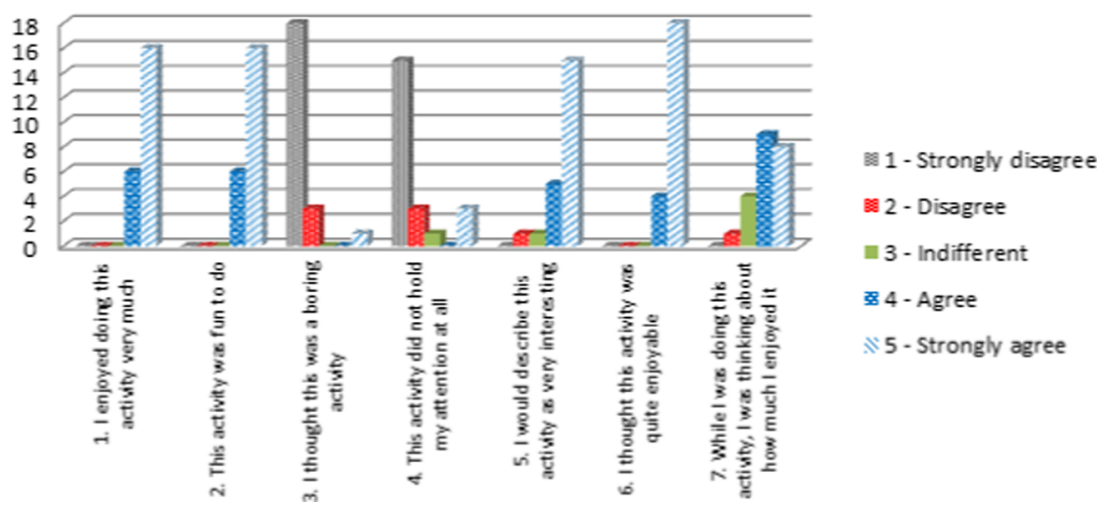


Fig. 5 IMI answers distribution online test

\section{IMI - online test}

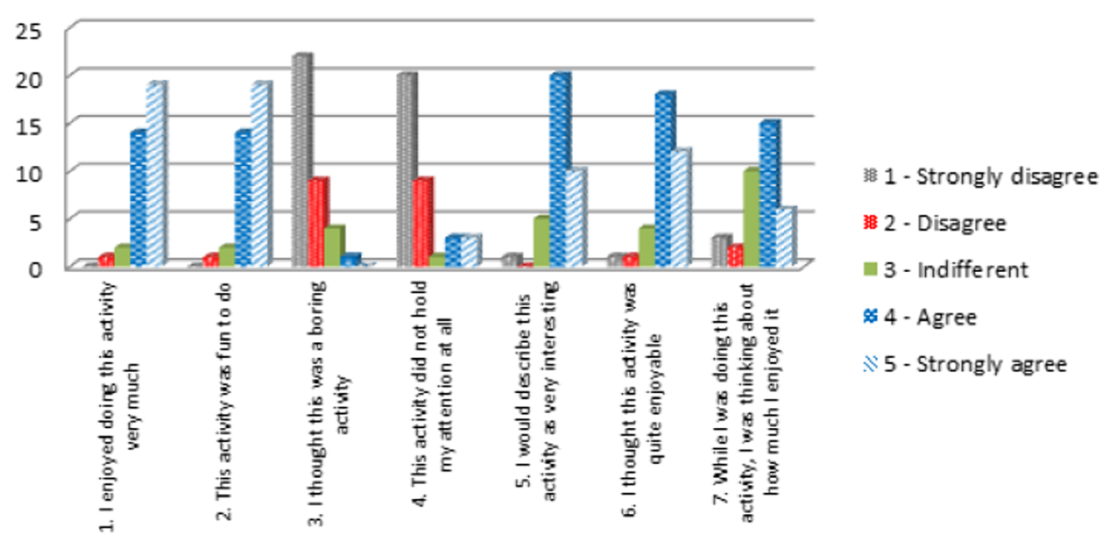

usability problems and more than 71.4 classifies the system as having good usability [91]. There were significant differences $(P=0.046<0.05)$ with respect to age $(<30$ years, $>30$ years $)$ of users, and the values of the questionnaire were significantly higher in participants with more than 30 years (39.78 vs. 27.61). The results observed in this study $(88.7 \%)$ indicate that the platform has a positive assessment in relation to its usability. More specifically, it can be stated that the platform offers good usability.

The SUS score results were also compared using the two independent samples. Table 4 presents the mean and standard deviation of the SUS score obtained by the classroom and online tests samples.

The mean value is higher in the classroom test (90.2\%), however still very good when the individuals did the test online $(87.1 \%)$. Applying the Mann-Whitney test ( $p$ value 0.45 ) there was not statistical evidences of the difference between results distribution of usability by making the test online and in the classroom.

Figures 2 and 3 show the SUS answers distribution about the classroom and online tests, respectively.

The answers distribution confirms the good level of concordance in terms of usability of the overall system.

It was used in this research one scale of the IMI questionnaire, to assess the interest and satisfaction of individuals in relation to all the contact they had with the Rehab+ platform (Table 4). The total result $(87.0 \%)$ shows that users were satisfied with the platform and that it aroused their interest. Most users answered that they would like to use this platform often $(74.2 \%)$ and $94.8 \%$ answered they liked to participate in this activity and that it was fun. The mean value of IMI score is also higher in the classroom test (90.9 \%), however still very good when the individuals did the test online $(83.0 \%)$. Applying the Mann-Whitney test ( $p$ value 0.015 ) reveals that there was statistical evidence of the difference between the IMI distribution results by playing online and in the classroom.
Figures 4 and 5 show the IMI answers distribution about the classroom and online tests, respectively.

The IMI answers distribution also reveals that most of individuals considered playing the games was very enjoyable and fun, regarding doing the tests online or in the classroom.

These results show the motivation and the interest of the participants by playing these games.

\section{Conclusions and future work}

The main objective of this work was make a preliminary study about the inclusion of a set of features that can contribute to innovate the cognitive therapy processes, making them more motivating for patients. To this aim we developed a web platform of Serious Games for cognitive rehabilitation that integrates social features such as cooperation, competition, and handicapping, and enables user interaction via mouse and voice commands. Some of the games can be played in multiplayer versions of two users. All versions can be played using the mouse and one version can be played using voice commands.

Tests were carried out on a sample of healthy subjects and without significant limitations. The usability of the platform was evaluated, resulting in a positive assessment. Also, it was assessed the interest and satisfaction of individuals in relation to all the contact they had with the Rehab+ platform and the total result showed that users were satisfied with the platform and that it aroused their interest. The distribution of users' answers revealed that most of them considered that playing the games was very enjoyable and fun. The main limitations presented in this work are related with the sample size and the population of the individuals, since they do not present any physical or cognitive limitation. However, for a preliminary assessment of the usability of a game that integrates social features such as cooperation, competition, and handicapping, and enables user interaction via mouse and voice commands, 
the results show a promising way for the rehabilitation of users.

For future work we intend to validate these games in a population with limitations, either physical or cognitive, and to participate effectively in a cognitive therapy process where we can assess the virtues of these games as well as natural interaction interfaces, such as voice, used here, which can show to be more useful and functional to someone with motor difficulties.

Acknowledgments This work has been supported by FCT - Fundação para a Ciência e Tecnologia in the scope of the projects: PEst-UID/CEC/ 00319/2015 and PEst-UID/CEC/00027/2015. The authors would like to thank also all the volunteers that participated in the study.

\section{References}

1. Burke, J. W., McNeill, M. D. J., Charles, D. K., Morrow, P. J., Crosbie, J. H., and McDonough, S. M., Optimising engagement for stroke rehabilitation using serious games. Vis. Comput. 25: 1085-1099, 2009.

2. Burke, J. W., McNeill, M. D. J., Charles, D. K., Morrow, P. J., Crosbie, J. H., McDonough, S. M. Augmented reality games for upper-limb stroke rehabilitation. In: 2010 second international conference on games and virtual worlds for serious applications (VSGAMES). pp. 75-78. 2010.

3. Maclean, N., Pound, P., Wolfe, C., and Rudd, A., Qualitative analysis of stroke patients' motivation for rehabilitation. $\mathrm{Br}$. Med. J. 321:1051-1054, 2000.

4. Krichevets, A. N., Sirotkina, E. B., Yevsevicheva, I. V., and Zeldin, L. M., Computer games as a means of movement rehabilitation. Disabil. Rehabil. 17:100-105, 1995.

5. Rego, P., Moreira, P. M., Reis, L. P., Serious games for rehabilitation: a survey and a classification towards a taxonomy. In: 5th Iberian conference on information systems and technologies. Vol. I. pp. 349-354. Santiago de Compostela, Spain, 2010.

6. Rego, P. A., Moreira, P. M., Reis, L. P., New forms of interaction in serious games for rehabilitation. In: Cruz-Cunha, M. M., (Ed.), Handbook of research on serious games as educational, business, and research tools: development and design. IGI Global, 2012.

7. Rego, P. A., Moreira, P. M., and Reis, L. P., A serious games framework for health rehabilitation. Int. J. Healthc. Inf. Syst. Inf. (IJHISI) 9:1-21, 2014.

8. Rego, P. A., Moreira, P. M., Reis, L. P., Architecture for serious games in health rehabilitation. In: Rocha, Á., Correia, A. M., Tan, F. B., Stroetmann, K. A.. (Eds.), New perspectives in information systems and technologies, volume 2, Vol. 276. pp. 307-317. Springer International Publishing, 2014.

9. Mendes, L., Dores, A. R., Rego, P. A., Moreira, P. M., Barbosa, F., Reis, L. P., Viana, J., Coelho, A., and Sousa, A., Virtual centre for the rehabilitation of road accident victims (VICERAVI). In: Rocha, A., CalvoManzano, J., Reis, L. P., and Cota, M. P. (Eds.), 7th Iberian conference on information systems and technologies (CISTI 2012), vol. I. AISTI, Madrid, pp. 817-822, 2012.

10. Rocha, R., Reis, L. P., Rego, P. A., Moreira, P. M., Serious games for cognitive rehabilitation: Forms of interaction and social dimension. In: 2015 10th Iberian conference on information systems and technologies (CISTI). pp. 1-6. 2015.

11. Alankus, G., Lazar, A., May, M., Kelleher, C., Towards customizable games for stroke rehabilitation. In: Proceedings of the SIGCHI conference on human factors in computing systems. pp. 2113-2122. ACM, Atlanta, Georgia, USA, 2010.

12. Ma, M., and Bechkoum, K., Serious games for movement therapy after stroke. IEEE international conference on systems, man and cybernetics. International Convention \& Exhibition Center, Suntec Singapore, pp. 1872-1877, 2008.

13. Karray, F., Alemzadeh, M., Saleh, J. A., and Arab, M. N., Humancomputer interaction: overview on state of the art. Int. J. Smart Sens. Intell. Syst. 1:137-159, 2008.

14. Oviatt, S., Multimodal interfaces. In: Julie, A. J., Andrew, S., (Eds.), The human-computer interaction handbook, pp. 286-304. L. Erlbaum Associates Inc, 2003.

15. Rego, P. A., Moreira, P. M., Reis, L. P., Natural user interfaces in serious games for rehabilitation: a prototype and playability study. In: Rocha, Á., Gonçalves, R., Cota, M. P., Reis, L. P., (Eds.), First Iberian Workshop on Serious Games and Meaningful Play (SGaMePlay'2011) - Proceedings of the 6th iberian conference on information systems and technologies, Vol. I. pp. 229-232. Chaves, Portugal, 2011.

16. Rego, P. A., Moreira, P. M., Reis, L. P., Natural and multimodal user interfaces in serious games for health rehabilitation. In: MASH'14: Multi-agent systems for healthcare / AAMAS'14 - 13th international conference on autonomous agents and multiagent systems. IFAMAAS, 2014.

17. Jaimes, A., and Sebe, N., Multimodal human-computer interaction: a survey. Comput. Vis. Image Underst. 108:116-134, 2007.

18. Jain, J., Lund, A., Wixon, D., The future of natural user interfaces. In: CHI '11 extended abstracts on human factors in computing systems. pp. 211-214. ACM, 1979527, 2011.

19. Chai, J. Y., Hong, P., Zhou, M. X., A probabilistic approach to reference resolution in multimodal user interfaces. In: Proceedings of the 9th international conference on intelligent user interfaces. pp. 70-77. ACM, Funchal, Madeira, Portugal, 2004.

20. Faria, B. M., Reis, L. P., Lau, N., Soares, J. C., and Vasconcelos, S., Patient classification and automatic configuration of an intelligent wheelchair. In: Filipe, J., and Fred, A. (Eds.), Agents and artificial intelligence, vol. 358. Springer, Berlin Heidelberg, pp. 268-282, 2013.

21. Johnston, M., Bangalore, S., MATCHkiosk: a multimodal interactive city guide. In: Proceedings of the ACL 2004 on Interactive poster and demonstration sessions. pp. 33. Association for Computational Linguistics, 2004.

22. Ibrahim, A., and Johansson, P., Multimodal dialogue systems: a case study for interactive TV. In: Carbonell, N., and Stephanidis, C. (Eds.), Universal access theoretical perspectives, practice, and experience: 7th ERCIM international workshop on user interfaces for all, Paris, France, October 24-25, 2002, revised papers. Springer Berlin Heidelberg, Berlin, pp. 209-218, 2003.

23. Morikawa, C., and Lyons, M. J., Design and evaluation of visionbased head and face tracking interfaces for assistive input. In: Georgios, K. (Ed.), Assistive technologies and computer access for motor disabilities. IGI Global, Hershey, pp. 180-205, 2014.

24. Ronzhin, A., Karpov, A., Assistive multimodal system based on speech recognition and head tracking. In: Proceedings of 13th European Signal Processing Conference. 2005

25. Reis, L., Faria, B., Vasconcelos, S., Lau, N., Invited paper: multimodal interface for an intelligent wheelchair. In: Ferrier, J.-L., Gusikhin, O., Madani, K., Sasiadek, J., (Eds.), Informatics in control, automation and robotics, Vol. 325. pp. 1-34. Springer International Publishing, 2015

26. Ogiela, M. R., and Hachaj, T., Natural user interfaces in medical image analysis: cognitive analysis of brain and carotid artery images. Springer International Publishing, Switzerland, 2014.

27. Steinberg, G., Natural user interfaces. In: ACM SIGCHI conference on human factors in computing systems. 2012. 
28. Faria, B. M., Reis, L. P., Lau, N., Moreira, A. P., Petry, M., Ferreira, L. M., Intelligent wheelchair driving: bridging the gap between virtual and real intelligent wheelchairs. In: Pereira, F., Machado, P., Costa, E., Cardoso, A., (Eds.), Progress in artificial intelligence. Vol. 9273, pp. 445-456. Springer International Publishing, 2015.

29. Faria, B. M., Reis, L. P., Lau, N., A methodology for creating an adapted command language for driving an intelligent wheelchair. J. Intell. Robot. Syst. 80, 2015.

30. Faria, B., Reis, L., and Lau, N., Adapted control methods for cerebral palsy users of an intelligent wheelchair. J. Intell. Robot. Syst. 77:299-312, 2015.

31. Faria, B. M., Silva, A., Faias, J., Reis, L. P., Lau, N., Intelligent wheelchair driving: a comparative study of cerebral palsy adults with distinct boccia experience. In: Rocha, Á., Correia, A. M., Tan, F. B., Stroetmann, K. A., (Eds.), New perspectives in information systems and technologies, volume 2. Vol. 276. pp. 329-340. Springer International Publishing, 2014.

32. Faria, B. M., Vasconcelos, S., and Reis, L. P., Evaluation of distinct input methods of an intelligent wheelchair in simulated and real environments: a performance and usability study. Assist. Technol. Off. J. RESNA 25:88-98, 2013.

33. Faria, B., Reis, L., Teixeira, S., Faias, J., Lau, N., Intelligent wheelchair simulator for users' training cerebral palsy children's case study. In: 8th Iberian conference on information systems and technologies (CISTI). 2013.

34. Faria, B. M., Vasconcelos, S., Reis, L. P., Lau, N., A methodology for creating intelligent wheelchair users' profiles. In: ICAART 2012 - 4th International conference on agents and artificial intelligence. pp. 171-179. 2012.

35. Moussa, M. B., Magnenat-Thalmann, N., Applying affect recognition in serious games: the playmancer project. In: Egges, A., Geraerts, R., Overmars, M., (Eds.), Motion in games. pp. 53-62. Springer, 2009.

36. Gerling, K., Livingston, I., Nacke, L., Mandryk, R., Full-body motion-based game interaction for older adults. In: Proceedings of the SIGCHI conference on human factors in computing systems. pp. 1873-1882. ACM, Austin, Texas, USA, 2012.

37. Chang, Y.-J., Chen, S.-F., and Chuang, A.-F., A gesture recognition system to transition autonomously through vocational tasks for individuals with cognitive impairments. Res. Dev. Disabil. 32:2064 2068, 2011.

38. Ciger, J., Herbeliny, B., Thalmannz, D., Evaluation of gaze tracking technology for social interaction in virtual environments. In: Proceedings of the 2nd workshop on modeling and motion capture techniques for virtual environments (CAPTECH04). 2004.

39. Jacob, R. J. K., Karn, K. S., Eye tracking in human-computer interaction and usability research: ready to deliver the promises. The mind's eye: cognitive the mind's eye: cognitive and applied aspects of eye movement research. pp. 573-603. 2003.

40. Mohamed, A. O., Silva, M. P. D., Courboulay, V., A history of eye gaze tracking. Tech. Rep. 2008.

41. Cowie, R., Douglas-Cowie, E., Tsapatsoulis, N., Votsis, G., Kollias, S., Fellenz, W., and Taylor, J. G., Emotion recognition in humancomputer interaction. IEEE Signal Process. Mag. 18:32-80, 2001.

42. Li, S. Z., and Jain, A. K., Handbook of face recognition. Springer Science \& Business Media, Germany, 2011.

43. Menache, A., Understanding motion capture for computer animation and video games. Morgan Kaufmann, 2000.

44. Kirishima, T., Sato, K., and Chihara, K., Real-time gesture recognition by learning and selective control of visual interest points. IEEE Trans. Pattern Anal. Mach. Intell. 27:351-364, 2005.

45. Gavrila, D. M., The visual analysis of human movement: a survey. Comput. Vis. Image Underst. 73:82-98, 1999.

46. Bradski, G. R., Computer vision face tracking for use in a perceptual user interface. In: Proceedings of the fourth IEEE workshop on applications of computer vision (WACV'98). 1998.
47. Wachs, J. P., Kölsch, M., Stern, H., and Edan, Y., Vision-based hand-gesture applications. Commun. ACM 54:60-71, 2011.

48. Microsoft kinect for Windows. Available: https://developer. microsoft.com/en-us/windows/kinect, 2016.

49. Leap motion. Available: https://www.leapmotion.com/ 2016.

50. Duchowski, A. T., A breadth-first survey of eye-tracking applications. Behav. Res. Methods Instrum. Comput. 34:455-470, 2002.

51. Duchowski, A., Eye tracking methodology: theory and practice. Springer Science \& Business Media, 2007.

52. Bulling, A., and Gellersen, H., Toward mobile Eye-based humancomputer interaction. IEEE Pervasive Comput. 9:8-12, 2010.

53. Dickie, C., Vertegaal, R., Sohn, C., Cheng, D., Eyelook: using attention to facilitate mobile media consumption. In: Proceedings of the 18th annual ACM symposium on user interface software and technology. pp. 103-106. ACM, Seattle, WA, USA, 2005.

54. Zhai, S., Morimoto, C., Ihde, S., Manual and gaze input cascaded (MAGIC) pointing. In: Proceedings of the SIGCHI conference on Human factors in computing systems: the CHI is the limit. pp. 246 253. ACM, Pittsburgh, Pennsylvania, United States, 1999.

55. Tobii. Available: http://www.tobii, 2015.

56. Schneiderman, R., Accuracy, apps advance speech recognition [special reports]. IEEE Signal Process. Mag. 32:12-125, 2015.

57. Schroeder, M. R., Computer speech: recognition, compression, synthesis. Springer Science \& Business Media, 2004.

58. Igarashi, T., Hughes, J. F., Voice as sound: using non-verbal voice input for interactive control. In: Proceedings of the 14th annual ACM symposium on User interface software and technology. pp. 155-156. ACM, Orlando, Florida, 2001.

59. Sporka, A. J., Kurniawan, S. H., and Slavík, P., Non-speech operated emulation of keyboard. In: Clarkson, J., Langdon, P., and Robinson, P. (Eds.), Designing accessible technology. Springer London, London, pp. 145-154, 2006.

60. Bilmes, J. A., Li, X., Malkin, J., Kilanski, K., Wright, R., Kirchhoff, K., Subramanya, A., Harada, S., Landay, J. A., Dowden, P., Chizeck, H., The vocal joystick: a voice-based human-computer interface for individuals with motor impairments. In: Proceedings of the conference on human language technology and empirical methods in natural language processing. pp. 995-1002. Association for Computational Linguistics, 2005.

61. Poláček, O., Sporka, A. J., and Míkovec, Z., Measuring performance of a predictive keyboard operated by humming. In: Miesenberger, K., Karshmer, A., Penaz, P., and Zagler, W. (Eds.), Computers helping people with special needs: 13th international conference, ICCHP 2012, Linz, Austria, July 11-13, 2012, proceedings, part II. Springer Berlin Heidelberg, Berlin, pp. 467-474, 2012.

62. Harada, S., Wobbrock, J. O., and Landay, J. A., Voice games: investigation into the use of Non-speech voice input for making computer games more accessible. In: Campos, P., Graham, N., Jorge, J., Nunes, N., Palanque, P., and Winckler, M. (Eds.), Human-computer interaction - INTERACT 2011: 13th IFIP TC 13 international conference, Lisbon, Portugal, September 5-9, 2011, proceedings, part I. Springer Berlin Heidelberg, Berlin, pp. 11-29, 2011.

63. Sporka, A. J., Kurniawan, S. H., Mahmud, M., Slavík, P., Nonspeech input and speech recognition for real-time control of computer games. In: Proceedings of the 8th international ACM SIGACCESS conference on computers and accessibility. pp. 213220. ACM, Portland, Oregon, USA, 2006.

64. Pierre-Yves, O., The production and recognition of emotions in speech: features and algorithms. Int. J. Hum. Comput. Stud. 59: 157-183, 2003.

65. Ververidis, D., and Kotropoulos, C., Emotional speech recognition: resources, features, and methods. Speech Comm. 48:1162-1181, 2006. 
66. Schiel, F., Steininger, S., Türk, U., The SmartKom multimodal corpus at BAS. In: Proc. 3rd Int. Conf. on Language Resources and Evaluation (LREC 2002). pp. 35-41. 2002.

67. France, D. J., Shiavi, R. G., Silverman, S., Silverman, M., and Wilkes, M., Acoustical properties of speech as indicators of depression and suicidal risk. IEEE Trans. Biomed. Eng. 47:829-837, 2000.

68. Ozdas, A., Shiavi, R. G., Silverman, S. E., Silverman, M. K., and Wilkes, D. M., Investigation of vocal jitter and glottal flow spectrum as possible cues for depression and near-term suicidal risk. IEEE Trans. Biomed. Eng. 51:1530-1540, 2004.

69. Schröder, M., Heylen, D., and Poggi, I., Perception of non-verbal emotional listener feedback. In: Hoffmann, R., and Mixdorff, H. (Eds.), Speech prosody 2006, vol. 40. TUDpress, Dresden, pp. 43-46, 2006.

70. Kostoulas, T., Mporas, I., Kocsis, O., Ganchev, T., Katsaounos, N., Santamaria, J. J., Jimenez-Murcia, S., Fernandez-Aranda, F., and Fakotakis, N., Affective speech interface in serious games for supporting therapy of mental disorders. Exp. Syst. Appl. 39: 11072-11079, 2012.

71. Hayward, V., Astley, O. R., Cruz-Hernandez, M., Grant, D., and Robles-De-La-Torre, G., Haptic interfaces and devices. Sens. Rev. 24:16-29, 2004.

72. Göger, D., Weiß, K., Burghart, C., Wörn, H., Sensitive skin for a humanoid robot. In: Proceedings of the 2006 international conference on human-centered robotic systems. 2006.

73. AAPB. Available: http://www.aapb.org/, 2011.

74. Conconi, A., Ganchev, T., Kocsis, O., Papadopoulos, G., Fernandez-Aranda, F., Jimenez-Murcia, S., PlayMancer: a serious gaming 3D environment. In: International conference on automated solutions for cross media content and multi-channel distribution (AXMEDIS '08). pp. 111-117. Institute of Electrical and Electronics Engineers (IEEE), 2008.

75. Nacke, L. E., Kalyn, M., Lough, C., Mandryk, R .L., Biofeedback game design: using direct and indirect physiological control to enhance game interaction. In: Proceedings of the SIGCHI Conference on Human Factors in Computing Systems. pp. 103-112. ACM, Vancouver, BC, Canada, 2011.

76. Kuikkaniemi, K., Laitinen, T., Turpeinen, M., Saari, T., Kosunen, I., Ravaja, N., The influence of implicit and explicit biofeedback in first-person shooter games. In: Proceedings of the SIGCHI conference on human factors in computing systems. pp. 859-868. ACM, Atlanta, Georgia, USA, 2010.

77. Flynn, S., Palma, P., and Bender, A., Feasibility of using the Sony PlayStation 2 gaming platform for an individual poststroke: a case report. J. Neurol. Phys. Ther. 31:180-189, 2007.

78. Saposnik, G., Teasell, R., Mamdani, M., Hall, J., McIlroy, W., Cheung, D., Thorpe, K., Cohen, L., and Bayley, M., Effectiveness of virtual reality using Wii gaming technology in stroke rehabilitation: a pilot randomized clinical trial and proof of principle. Stroke 41:1477-1484, 2010.

79. Nintendo: Wii console. Available: http://www.nintendo. com/wii/console, 2014

80. Sony: playstation move. Available: http://pt.playstation. com/psmove/, 2014.

81. Vanacken, L., Notelaers, S., Raymaekers, C., Coninx, K., van den Hoogen, W., Jsselsteijn, W. I., Feys, P., Game-based collaborative training for arm rehabilitation of MS patients: a proof-of-concept game. In: Proceedings of the GameDays 2010. pp. 65-75. 2010.

82. Battocchi, A., Gal, E., Ben Sasson, A., Painesi, F., Venuti, P., Zancanaro, M., Weiss, P. L., Collaborative puzzle game - an interface for studying collaboration and social interaction for children who are typically developed or who have autistic spectrum disorder. In: Proceedings of the 7th International Conference series on disability, virtual reality and associated technologies (ICDVRAT). pp. 127-134. 2008.

83. Battocchi, A., Pianesi, F., Tomasini, D., Zancanaro, M., Esposito, G., Venuti, P., Sasson, A. B., Gal, E., Weiss, P. L., Collaborative puzzle game: a tabletop interactive game for fostering collaboration in children with Autism Spectrum Disorders (ASD). In: Proceedings of the ACM international conference on interactive tabletops and surfaces. pp. 197-204. ACM, Banff, Alberta, Canada, 2009.

84. Caglio, M., Latini-Corazzini, L., D'agata, F., Cauda, F., Sacco, K., Monteverdi, S., Zettin, M., Duca, S., and Geminiani, G., Video game play changes spatial and verbal memory: rehabilitation of a single case with traumatic brain injury. Cogn. Process. 10:195-197, 2009.

85. Cameirão, M. S., Badia, S. B., Zimmerli, L., Oller, E. D., and Vershure, P. F. M. J., The rehabilitation gaming system: a review. Stud. Health Technol. Inform. 145:65-83, 2009.

86. Hasomed RehaCom. Available: https://www.hasomed. $\mathrm{de} / \mathrm{en} / \mathrm{rehacom} /$ what-is-rehacom/product-overview.html?L=2, 2016.

87. Maia, L., Gaspar, C., Azevedo, M., Loureiro, M. J., and Silva, C. F., Reabilitação cognitiva assistida por computador: o programa RehaCom e a sua utilização no GEARNeurop. Psiquiatr. Clín. 25: 83-105, 2004.

88. Parrot software. Available: http://www.parrotsoftware.com/, 2016.

89. Fundación intras. Available: http://www.intras.es/index.php?id=75, 2014.

90. StatCounter: GlobalStats. Available: http://gs.statcounter. com/\#browser-ww-monthly-201409-201509-bar, 2015.

91. Bangor, A., Kortum, P., and Miller, J., Determining what individual SUS scores mean: adding an adjective rating scale. J. Usability Stud. 4:114-123, 2009. 